\title{
Prevalencia del síndrome de burnout en la dirección de instituciones educativas públicas en Barrios Altos, Lima - Perú
}

\author{
Prevalence of Burnout Syndrome in Principals of Public Educational Institutions in Barrios \\ Altos, Lima, Perú
}

\section{Prevalência da Síndrome de Burnout na gestão escolar de instituições públicas de ensino em Barrios Altos, Lima - Perú}

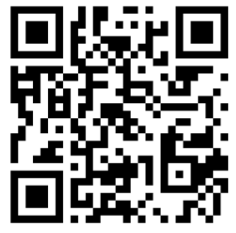

Juan Pablo De la Guerra-De Urioste Universidad San Ignacio de Loyola

Lima, Perú jpdelaguerra@gmail.com

iD https://orcid.org/0000-0001-6259-8755

Deyanira Aneel Goicochea-Rojas Municipalidad Metropolitana de Lima Lima, Perú

deyaniraa.goicochear@gmail.com https://orcid.org/0000-0002-4300-316X

Pablo César Torres-Cañizalez Universidad César Vallejo Lima, Perú ptorresca17@ucvvirtual.edu.pe https://orcid.org/0000-0001-9570-4526

John Kendry Cobo-Beltrán Universidad César Vallejo Lima, Perú jcobobe@ucvvirtual.edu.pe https://orcid.org/0000-0003-0997-3821

Recibido • Received • Recebido: 27 / 12 / 2019

Corregido • Revised • Revisado: 19 / 07 / 2021

Aceptado • Accepted • Aprovado: 23 / 08 / 2021 
http://doi.org/10.15359/ree.25-3.28

http://www.una.ac.cr/educare

educare@una.ac.cr

\begin{abstract}
Resumen:
Objetivo. Esta investigación tiene como propósito determinar la prevalencia del síndrome de burnout en la dirección escolar de las instituciones de educación básica regular en la zona de Barrios Altos, Lima. Metodología. Se empleó un diseño de investigación no experimental, con una orientación descriptiva del evento de estudio, para lo cual se aplicó el Maslach Burnout Inventory a los 22 sujetos directores que conforman el equipo de dirección de las instituciones educativas públicas, que constituyen el $100 \%$ de la población del estudio. Resultados. Se ha encontrado un nivel medio de agotamiento emocional, un bajo nivel de despersonalización y alta realización personal. Conclusiones. La prevalencia del síndrome de burnout en el colectivo de los sujetos directores es baja; sin embargo, se encontraron niveles moderados de agotamiento emocional, los cuales constituyen un rasgo que, de mantenerse en el tiempo, podría derivar en burnout. Este trabajo constituye un aporte investigativo preliminar, que deja el camino abierto para futuros estudios relacionados con el personal directivo escolar y el síndrome de burnout.
\end{abstract}

Palabrasclave:Síndromede Burnout, agotamientoemocional, realización personal, despersonalización, estrés, dirección escolar.

\begin{abstract}
:
Objective. This research aims to determine the prevalence of burnout syndrome in principals of regular basic education institutions in Barrios Altos, Lima. Method. A non-experimental research design was used, with a descriptive orientation of the study event, for which the Maslach Burnout Inventory was applied to 22 principals from a management team of public educational institutions, which constitute $100 \%$ of the study population. Results. A medium level of emotional exhaustion, a low level of depersonalization, and highly personal accomplishment have been found. Conclusions. The prevalence of burnout syndrome in the group of principals is low, but moderate levels of exhaustion are found emotional, which are a feature that, if maintained over time, could lead to burnout. This work constitutes a preliminary research contribution, leaving the way for future studies related to school management and burnout syndrome.
\end{abstract}

Keywords: Burnout syndrome; emotional exhaustion; personal fulfillment; depersonalization; stress; school management.

\title{
Resumo:
}

Objetivo. Esta pesquisa tem como objetivo determinar a prevalência da síndrome de Burnout na gestão escolar de instituições de educação básica na área de Barrios Altos, Lima. Metodologia. Utilizou-se um delineamento de pesquisa não experimental, com orientação descritiva do evento de estudo, para o qual o Maslach Burnout Inventory foi aplicado aos 22 diretores que compõem a equipe de gestão das instituições públicas de ensino, que constituem $100 \%$ da população do estudo. Resultado. Foi encontrado um nível médio de exaustão emocional, baixo nível de despersonalização e alta realização pessoal. Conclusão. A prevalência da síndrome de Burnout no grupo de diretores é baixa, mas são encontrados níveis moderados de exaustão emocional, que é uma característica que, se mantida ao longo do tempo, pode levar ao esgotamento. Este trabalho constitui uma contribuição de pesquisa preliminar, que abre caminho para futuros estudos relacionados à gestão escolar e à síndrome de Burnout.

Palavraschave:SíndromedeBurnout;esgotamentoemocional;satisfação pessoal;despersonalização; estresse; gestão escolar. 


\section{Introducción}

Desde hace más de catorce años Freudenberguer (1974) denominó como "Burnout" al desgaste físico y psicológico que produce una sobrecarga de trabajo estresante, sostenida en el tiempo y no manejada de manera adecuada. Con el paso de los años, el síndrome de burnout ha sido objeto de una enorme cantidad de estudios y se ha posicionado en los ámbitos organizacionales como una variable a tener en cuenta en sus contextos (Tejero González et al., 2010).

En este sentido, el referente teórico que ha tenido un mayor impacto en el estudio del síndrome de burnout es la psicóloga norteamericana Christina Maslach. De acuerdo con Maslach (2017), este síndrome constituye una respuesta orgánica ante el estrés laboral que muestran únicamente quienes se dedican a labores asistenciales en la que existen diversas presiones y requerimientos por parte de grupos humanos, como, por ejemplo, docentes, personal de salud o de voluntariado. Sobre estos grupos profesionales orientados hacia el servicio social existe una gran cantidad de estudios, sin embargo, en cuanto a directores y directoras escolares no es posible encontrar la misma abundancia de trabajos.

La dirección escolar es una de las labores profesionales con mayor riesgo de sufrir las consecuencias del estrés laboral, debido a la reconfiguración de las funciones, responsabilidades y tareas asignadas a quien ejerza la dirección de la institución educativa (Ramery-Gelpi y Pérez Navío, 2016). A dicha función se suman las crecientes demandas sociales acerca de la responsabilidad que se le atribuye al personal directivo sobre el éxito o fracaso de todos los procesos que se generan en el centro educativo (Alsalamah y Callinan, 2021), y a la complejización de los contextos sociales y comunitarios en los cuales se ejerce la función directiva.

Por ello, esta investigación tiene como propósito determinar la prevalencia del síndrome de burnout en la dirección escolar de las instituciones de educación básica regular en Barrios Altos, Lima-Perú, a través de la aplicación del cuestionario Maslach Burnout Inventory a la totalidad de profesionales que desempeñan el cargo de director o directora. Es importante destacar que Barrios Altos es un emblemático espacio territorial enclavado en el centro histórico de la ciudad, en el cual convergen múltiples problemas sociales asociados a la pobreza, que repercuten directamente en la dinámica escolar $y$, por ende, en las condiciones laborales del sector educativo, por lo que, este estudio busca conocer, de forma científica, hasta qué punto esta población directiva está padeciendo los síntomas que genera la exposición a factores de estrés prolongado, con miras a visibilizar la situación de este colectivo profesional, promover las acciones necesarias para atenuar los estresores, y realizar un mejor manejo de la presión que supone ejercer la función directiva en contextos de vulnerabilidad social.

\section{Estado de la cuestión}

El síndrome de burnout ha sido motivo de estudio en distintos ámbitos del mundo laboral desde hace más de tres décadas en diferentes contextos ocupacionales, incluyendo el educativo, 
http://doi.org/10.15359/ree.25-3.28

http://www.una.ac.cr/educare

educare@una.ac.cr

con mayor énfasis en el personal docente. En el campo del burnout, en la dirección escolar los trabajos son relativamente pocos, pudiéndose citar con fines ilustrativos los trabajos de Chaplain (2001), Cooper y Kelly (1993), Friedman (1995), Hakan (2004), Lainas (2010), Olayiwola (2008), Ramery-Gelpi y Pérez Navío (2016), Tejero González et al. (2010), Tahira y Afshan (2019), Xu y Yang (2021) y Yusof (2012). Estos estudios coinciden en destacar los crecientes niveles de desgaste a los que se enfrentan los directores y las directoras escolares, así como también en afirmar que el síndrome de burnout es una respuesta al estrés crónico, al cual ha estado sometido el sujeto profesional que ejerce la dirección de la institución educativa.

\section{Referentes conceptuales}

El burnout syndrome, término acuñado por Freudenberger (1974), se traduce al castellano como síndrome de burnout, sin embargo, en la bibliografía se encuentran otras denominaciones equivalentes: síndrome de agotamiento profesional, desgaste profesional, estrés laboral asistencial o de quemarse por el trabajo. El síndrome de burnout es una respuesta al estrés laboral crónico (Maslach, 1976; Maslach, 2017; Maslach et al., 2001; Maslach y Jackson, 1981). En tal sentido, el síndrome de burnout presenta una sintomatología característica, con un alto componente emocional, la cual se menciona más adelante.

Debido a las consecuencias para la salud física y mental del individuo, y sus repercusiones en los entornos laborales y familiares, es importante identificar las diferencias existentes entre el burnout y otras afecciones psicológicas relacionadas, tales como la fatiga, el cansancio o la ansiedad, que surgen cuando el clima laboral no es el más sano (Gil-Monte, 2003), y pudieran tener un carácter transitorio. En el síndrome de burnout se suscitan un conjunto de síntomas sostenidos en el tiempo, organizados en tres componentes:

- Cansancio emocional: cansancio físico o mental, o una combinación de ambos, la persona se siente estar al límite de sus capacidades (Freudenberger, 1974; Gil-Monte, 2006; Maslach y Jackson, 1981).

- Despersonalización (cinismo): trato frío, hostil e irónico, incremento en la irritabilidad hacia clientes y compañeros y compañeras de trabajo (Golembiewski et al., 1993; Maslach et al., 2001).

- Falta de realización personal: actitudes negativas hacia sí y hacia el trabajo, incapacidad de soportar la presión y baja autoestima (Gil-Monte, 2006; Gil-Monte y Peiró, 1997; Maslach et al., 2001).

Con base en estos componentes, Maslach y Jackson (1981) desarrollaron el Maslach Burnout Inventory, el cual permite determinar la presencia del síndrome de burnout en un grupo o colectivo específico, y que goza de una alta aceptación de la comunidad científica, dadas sus 
http://doi.org/10.15359/ree.25-3.28

propiedades psicométricas (Gil-Monte, 2002; Hederich-Martínez y Caballero-Domínguez, 2016; Kulakova et al., 2017). Este instrumento ha sido aplicado a diversos colectivos profesionales, con ligeras adaptaciones para adecuarlas al contexto en el cual se utiliza (Maslach et al., 2001).

En la bibliografía se considera que el punto de origen del síndrome de burnout se encuentra en unas elevadas demandas laborales (Lee y Ashforth, 1996), sin embargo, identificar con exactitud sus factores desencadenantes constituye una tarea compleja, debido a que cualquier situación recurrente en el entorno laboral puede incidir en la aparición del síndrome (GilMonte y Peiró, 1997). El síndrome de burnout surge como resultado de una fuerte y prolongada exposición a estresores, es decir, a estímulos aversivos que, tras su percepción y evaluación, producen una respuesta (Gil-Monte, 2003).

En este sentido, el contexto organizacional, que incluye la relación interpersonal con clientes o público usuario, así como con colegas, personal de jefatura y subalterno, constituye un factor desencadenante (Gil-Monte, 2006); mientras que las características personales expresadas en la personalidad, el carácter y el concepto de sí cumplen un papel catalizador o inhibidor del síndrome (Maslach, 2017). Una vez que se manifiesta el burnout, la persona afectada manifiesta una serie de comportamientos adversos, tanto en el ámbito organizacional (insatisfacción laboral, ausentismo laboral, ineficiencia), como en el plano familiar y personal (agotamiento físico y psicológico, irritación, conflictos familiares y de pareja).

En cuanto a las instituciones educativas, la dirección escolar es considerada como un factor de primer orden para la calidad formativa, por estar involucrada en todos los procesos que se realizan en la escuela (Alsalamah y Callinan, 2021). Existe un claro consenso en asociar el liderazgo pedagógico que tenga el ente director con la calidad de la enseñanza y el aprendizaje del alumnado (Fullan, 2014), con la planeación estratégica y establecimiento de objetivos (Veenman, 1997), con la motivación del personal y el desarrollo del potencial del profesorado (Bolívar, 2015; Martínez-Garrido, 2017), con la creación de un clima escolar positivo y de satisfacción laboral (Ramery-Gelpi y Pérez Navío, 2016; Tejero González et al., 2010), con la evaluación de procesos y el acompañamiento (Bolívar, 2015). En líneas generales, a la dirección escolar se la atribuye la responsabilidad de la calidad educativa, la innovación docente, el rendimiento del centro y el éxito escolar.

Constatar la relevancia que se le otorga a la dirección de instituciones educativas supone identificar la diversidad de funciones, responsabilidades y tareas que recaen sobre la figura del director o directora, en contextos organizacionales y sociales cada vez más complejos (Alsalamah y Callinan, 2021). Estas funciones, a juicio de Barroso (2005), tienen un carácter híbrido, ambiguo y complejo. Entre los mayores retos para quien desempeña un cargo de dirección escolar es congeniar las funciones de gestión administrativa (planificación, organización, coordinación y control), que tradicionalmente habían sido el foco de atención; con la demanda emergente de constituirse en el líder pedagógico, promotor o promotora de la transformación profesional y personal de su equipo, y, además, ejercer el liderazgo comunitario (Bolívar, 2015). Debatirse 
http://doi.org/10.15359/ree.25-3.28

http://www.una.ac.cr/educare

educare@una.ac.cr

entre el papel administrativo, por una parte, y el de líder, por la otra, es un claro indicador de la dualidad funcional del personal directivo escolar (Louis y Miles, 1991), que lo posiciona en la jefatura ejecutiva y a su vez en el liderazgo profesional (Fullan, 2014; Hughes, 1987).

Además de la dualidad de funciones y la enorme responsabilidad inherente al ejercicio de la dirección escolar, se le suman un conjunto de situaciones adversas con las que debe lidiar el director o directora, entre las que destacan: la asfixiante burocracia, las limitaciones económicas, los frecuentes cambios en la política educativa y el currículo, la conflictividad de los padres y madres de familia, una autoridad limitada e insuficiente (Sáenz Barrio y Debón Lamarque, 1995). Desde este panorama, y desde hace décadas, los estudios colocan a la función directiva como una actividad de alto riesgo para la salud física y mental (Barroso, 2005).

En este sentido, la sumatoria de funciones, responsabilidades y tareas inherentes a la dirección, aunado al clima organizacional, hacen probable la exposición a estresores, que sostenidos en el tiempo pueden volverse estados crónicos desencadenantes del síndrome de burnout. Como forma de autoprotección ante situaciones altamente estresantes, a juicio de Sáenz Barrio y Debón Lamarque (1995), los grupos docentes en función directiva tienden a adoptar mecanismos de huida y de evitación. Según estos estudios, en la huida, los directores y las directoras hacen esfuerzos claros para escapar de la situación que supera sus posibilidades: traslado de la institución educativa, comisiones de servicio, licencia o descanso médico o renuncia a la función directiva; si optan por la evitación, entonces esquivan y burlan por el encuentro potencial con la situación problemática: gestiones fuera del plantel, enfermedades o problemas familiares fingidos o reales

En los casos en los cuales los mecanismos defensivos no han resultado efectivos, y las propias características psicológicas de quien ejerce la función directiva le hacen una persona propensa al estrés crónico, comienza a desencadenarse un proceso que va desde estadíos iniciales o de sintomatología leve, hasta llegar a estadíos finales o patológicos del síndrome de burnout (Chaplain, 2001; Cooper y Kelly, 1993; Friedman, 1995).

\section{Metodología}

\section{Criterios metodológicos}

La investigación es de tipo no experimental, y se asume desde una perspectiva descriptiva, debido a que se busca determinar la prevalencia del síndrome de burnout en la dirección escolar de Barrios Altos, sin intervenir, ni manipular variables; y para lograr ese cometido se aplicó el Maslach Burnout Inventory a la totalidad de los 22 directores y directoras de las instituciones educativas públicas. En este particular, la investigación asume características del estudio de casos, debido a que está estudiando a un colectivo completo, ubicado en un espacio territorial común, cuyos integrantes están inmersos en el mismo contexto socioeconómico, y regidos por la misma normativa. 
http://doi.org/10.15359/ree.25-3.28

\section{Unidades de estudio}

El estudio se realizó en las instituciones educativas públicas de Barrios Altos, que constituye una zona de Lima conformada por un conjunto de barrios tradicionales, y forma parte del casco histórico de la ciudad. Estas instituciones pertenecen a la Unidad de Gestión Escolar Local N. ${ }^{\circ}$ 3, de la Dirección Regional de Educación de Lima Metropolitana, organismos encargados de supervisar y hacer seguimiento a la gestión de las direcciones escolares de cada plantel.

\section{Sujetos participantes}

Las personas informantes en este estudio, a quienes se les aplicó el cuestionario Maslach Burnout Inventory, son los 22 directores y directoras de instituciones públicas de educación básica regular (inicial, primaria y secundaria), de Barrios Altos, pertenecientes al distrito de Lima Cercado del Perú. La muestra está conformada por el 100\% de la población en estudio; de los cuales 14 son mujeres $(63,6 \%)$ y 8 son hombres (36,4\%). El promedio de edades de este grupo de profesionales de la educación se encuentra entre los 46 y 65 años (72,8\%), con experiencia en el ejercicio del cargo desde 1 a 10 años (77,3\%); y en su gran mayoría cuentan con estudios de postgrado (Maestría, 72,7\%; Doctorado, 9,1\%; Especialización, 9,1\%).

\section{Procedimiento}

Para llevar a cabo esta investigación, se tomó como instrumento de recolección de datos el cuestionario Maslach Burnout Inventory (Maslach y Jackson, 1981). Se diseñó un formulario en Google con los ítems del instrumento, antecedido por algunas preguntas que permitieron categorizar los sujetos participantes (sexo, edades, grado académico, y años de experiencia).

Para la aplicación del instrumento se redactó un texto contentivo de las instrucciones para responder el cuestionario, con las debidas garantías de anonimato y confidencialidad de la información suministrada. Una vez que se constató que el 100\% de directores y directoras completaron el instrumento, se realizó el análisis estadístico de los datos, empleando el programa "Statistical Package for Social Science" (SPSS-22).

\section{Resultados}

En primera instancia se formulan algunas consideraciones técnicas acerca del instrumento utilizado en el estudio: el Maslach Burnout Inventory. Se realizó un análisis de cada una de las dimensiones del síndrome de burnout (agotamiento emocional, despersonalización, realización personal) y la prevalencia de ellas en los directores y las directoras de las instituciones educativas públicas de la zona de Barrios Altos, Lima. 
http://doi.org/10.15359/ree.25-3.28

http://www.una.ac.cr/educare

educare@una.ac.cr

Sobre esta base se realizó un análisis descriptivo de las variables sociolaborales de los sujetos (sexo, edad, años de experiencia en la función directiva, y máximo grado académico obtenido), en relación con las dimensiones del instrumento, para medir una posible asociación entre ellas. Todo este análisis permitió determinar los niveles de burnout del personal que desempeña funciones de dirección escolar.

\section{Acerca del instrumento}

El Maslach Burnout Inventory (MBI) (Maslach y Jackson, 1981, 1986), en su versión inicial para profesionales asistenciales y de servicio (Freudenberger, 1974; Maslach, 1976), está conformado por 22 ítems asociados a emociones, actitudes y sentimientos, los cuales, a su vez, estaban asociados hacia los sujetos receptores del servicio, compañeros y compañeras o hacia el trabajo. Estos se valoran con una escala tipo Likert con una frecuencia que va de $0=$ nunca a 6 = todos los días. La factorización de los ítems del MBI - HSS (Human Services Survey), aplicados a diversas muestras de profesionales con el MBI confirman la existencia de las tres dimensiones del síndrome de burnout: cansancio emocional (9 ítems), despersonalización (5 ítems) y realización personal (8 ítems).

Al aplicar el $\mathrm{MBI}$, se puede confirmar la presencia del síndrome de burnout cuando aparece un patrón de puntuaciones altas en las dimensiones de agotamiento emocional y despersonalización, pero bajas en la de realización personal. En este sentido, las puntuaciones máximas en las dimensiones del burnout son: agotamiento emocional, 54 puntos; despersonalización, 30 puntos; realización personal, 48 (Cooper y Kelly, 1993; Maslach y Jackson, 1981). En este sentido, los sujetos directivos cuyas puntuaciones sean superiores al percentil 75 se ubican en un nivel de "alto", entre el percentil 75 y el 25 en la categoría "medio"y, si están por debajo del percentil 25, en un nivel "bajo" (Maslach y Jackson, 1986). En la Tabla 1 se resumen las dimensiones del burnout y sus puntuaciones máximas referenciales.

Tabla 1: Dimensiones del síndrome de burnout y puntajes de referencia

\begin{tabular}{lccc}
\hline \multirow{2}{*}{ Niveles } & \multicolumn{3}{c}{ Puntajes en las dimensiones del síndrome de burnout } \\
\cline { 2 - 4 } & Cansancio emocional & Despersonalización & Realización personal \\
\hline Bajo $\left(<\mathrm{P}_{25}\right)$ & $<13,5$ & $<7,5$ & $<12$ \\
Medio $\left(\mathrm{P}_{25-\mathrm{P} 75}\right)$ & $13.5-40,5$ & $7,5-22,5$ & $12-36$ \\
Alto $\left(>\mathrm{P}_{75}\right)$ & $>40,5$ & $>22,5$ & $>36$ \\
\hline
\end{tabular}

Nota: Elaboración propia, basada en Maslach y Jackson (1986).

8 Juan Pablo De la Guerra-De Urioste, Deyanira Aneel Goicochea-Rojas, Pablo César Torres-Cañizalez y John Kendry Cobo-Beltrán

Los artículos de la Revista Electrónica Educare del Centro de Investigación y Docencia en Educación de la Universidad Nacional, Costa Rica, se comparten bajo términos de la Licencia Creative Commons: Reconocimiento, No Comercial, Sin Obra Derivada 3.0 Costa Rica. Las autorizaciones adicionales a las aquí delimitadas se pueden obtener en el correo: educare@una.cr 
http://doi.org/10.15359/ree.25-3.28

En cuanto a la validez y confiabilidad del instrumento empleado, el MBI-HSS es un instrumento validado y utilizado en una enorme diversidad de colectivos profesionales vinculados a la prestación de servicios. La consistencia interna del instrumento en uno de los estudios originales de Maslach y Jackson (1986) se calculó a partir de una muestra de 1316, mediante el alfa de Cronbach, y se obtuvieron coeficientes de 0,90 para la dimensión cansancio emocional; 0,79 en la de despersonalización; y de 0,71 en la de realización personal.

Para efectos de este estudio orientado a determinar la prevalencia del síndrome de burnout en la dirección de instituciones educativas públicas en Barrios Altos, Lima - Perú, se calculó la consistencia interna a través del alfa de Cronbach empleando el programa SPSS-22, el cual arrojó 0,908, lo cual indica una muy buena fiabilidad de la escala. Para obtener este valor fue necesario invertir los valores de los ítems 4, 7, 9, 12, 17 y 18 que inicialmente tenían una correlación negativa (ítems invertidos).

Por otra parte, es necesario e importante acotar que el Maslach Burnout Inventory ha recibido críticas. En este sentido, los datos obtenidos en diferentes culturas no pueden ser generalizados y asumirse como universales, pues si se pasan por alto los marcos conceptuales y culturales, se producirían insoslayables sesgos (Golembiewski et al., 1993). En atención a esto, es valioso el ajuste o adecuación de ciertos criterios del instrumento para obtener resultados más fidedignos (Gil-Monte, 2002). Aun así, el Maslach Burnout Inventory, desde 1981, se posiciona como la herramienta psicométrica más aceptada para determinar la prevalencia del síndrome de burnout, y se observa un sostenido apoyo empírico de estudios en colectivos de diversas dedicaciones ocupacionales.

\section{Análisis de las relaciones entre las dimensiones del síndrome}

El síndrome de burnout, en tanto respuesta a los estresores a los que se expone el sujeto en el ambiente laboral, tiene una condición tridimensional. A continuación, se analizan las dimensiones o componentes del burnout en la dirección escolar de la zona de Barrios Altos, Lima.

\section{a) Cansancio emocional}

Se refiere a la percepción de agotamiento emocional junto a una disminución de los recursos psicoafectivos de una persona al hacerle frente a las situaciones cotidianas, lo cual se acompaña de manifestaciones somáticas y psicológicas (Maslach y Jackson, 1981). Este componente del Maslach Burnout Inventory se evalúa mediante los ítems 1-2-3-6-8-13-14-1620. En la Tabla 2 se muestran las puntuaciones medias obtenidas por el personal que realiza funciones de dirección escolar de la zona de Barrios Altos en la subescala cansancio emocional. 
http://doi.org/10.15359/ree.25-3.28

http://www.una.ac.cr/educare

educare@una.ac.cr

Tabla 2: Cansancio emocional en la dirección escolar de la zona de Barrios Altos

\begin{tabular}{lcrc}
\hline Nivel & Rango & F & $\%$ \\
\hline Bajo & $(<13,5)$ & 5 & 22,7 \\
Medio & $(13,5-40,5)$ & 17 & 77,3 \\
Alto & $(>40,5)$ & 0 & 0,0 \\
\hline Total & - & 22 & 100 \\
\hline
\end{tabular}

Nota: Elaboración propia.

El cansancio emocional es considerado por diversos estudios como la dimensión que tiene mayor capacidad predictiva de la prevalencia del síndrome de burnout (Lee y Ashforth, 1996; Maslach, 2017; Maslach et al., 2001; Maslach y Jackson, 1986). En el caso de los directores y directoras de Barrios Altos, 17 de 22 (77,3\%) han arrojado puntuaciones que dan cuenta de un nivel medio de agotamiento emocional, factor que constituye un fuerte predictor de la prevalencia del síndrome.

\section{b) Despersonalización}

Valora el grado en que cada individuo reconoce en sí mismo actitudes de frialdad y distanciamiento (Golembiewski et al., 1993; Maslach et al., 2001). La despersonalización alude al endurecimiento emocional que muestra el individuo hacia las personas con la cuales interactúa cotidianamente en el mundo del trabajo. En el Maslach Burnout Inventory este componente se mide a través de los ítems: 5-10-11-15-22. Para el caso de docentes que ejercen la dirección escolar en Barrios Altos, en cuanto a la dimensión despersonalización en la Tabla 3 se presentan los resultados.

Tabla 3: Despersonalización en la dirección escolar de la zona de Barrios Altos

\begin{tabular}{lccc}
\hline Nivel & Rango & $\mathrm{f}$ & $\%$ \\
\hline Bajo & $<7,5$ & 14 & 63,6 \\
Medio & $7,5-22,5$ & 8 & 36,4 \\
Alto & $>22,5$ & 0 & 0,0 \\
\hline Total & - & 22 & 100 \\
\hline
\end{tabular}

Nota: Elaboración propia.

De acuerdo con estos datos, el equipo de dirección de los planteles escolares públicos de la zona de Barrios Altos mayoritariamente muestra una baja tendencia hacia la despersonalización, debido a que 14 personas obtuvieron puntuaciones que oscilan en el rango bajo (63,6\%), mientras que el resto del colectivo reporta una prevalencia media (36,4\%). 
http://doi.org/10.15359/ree.25-3.28

\section{C) Realización personal}

El componente realización personal del Maslach Burnout Inventory evalúa los sentimientos de realización personal y percepción de la propia eficiencia en el trabajo (GilMonte, 2006; Gil-Monte y Peiró, 1997; Maslach et al., 2001). Un nivel bajo denota que la persona se siente poco desarrollada laboralmente, sin entusiasmo y satisfacción por su trabajo, y es un importante predictor del burnout. La subescala del Maslach Burnout Inventory que determina el componente está integrada por los ítems 4-7-9-12-17-18-19-21. En la Tabla 4 se presenta el resultado en relación al componente realización personal.

Tabla 4: Realización Personal en la dirección escolar de la zona de Barrios Altos

\begin{tabular}{lccc}
\hline Nivel & Rango & $\mathrm{f}$ & $\%$ \\
\hline Bajo & $<12$ & 0 & 0,0 \\
Medio & $12-36$ & 9 & 40,9 \\
Alto & $>36$ & 13 & 59,1 \\
\hline Total & - & 22 & 100 \\
\hline
\end{tabular}

Nota: Elaboración propia.

De acuerdo con las respuestas emitidas por el personal en funciones de dirección de los planteles oficiales de la zona de Barrios Altos, existen fuertes indicios de autopercepción de realización personal, pues en los reactivos de la subescala, 13 personas directivas (59,1\%) alcanzaron puntuaciones que superan los 36 puntos, y no se observa ningún caso ubicado en el nivel bajo.

Una vez analizadas por separado cada una de las dimensiones del síndrome, medidas a través del Maslach Burnout Inventory, se presentan en la Tabla 5 los principales rasgos psicométricos de cada uno de los componentes estudiados.

Tabla 5: Tendencias dominantes de los componentes del síndrome en el personal directivo

\begin{tabular}{lccc}
\hline Componente & Nivel predominante & $\mathrm{f}$ & $\%$ \\
\hline Cansancio emocional & Medio & 17 & 54,5 \\
Despersonalización & Bajo & 14 & 63,6 \\
\hline Realización personal & Alto & 13 & 59,1 \\
\hline
\end{tabular}

Nota: Elaboración propia. 
http://doi.org/10.15359/ree.25-3.28

http://www.una.ac.cr/educare

educare@una.ac.cr

Estos resultados constituyen un indicio de que el personal directivo de las instituciones educativas públicas de Barrios Altos no está siendo afectado por el burnout en el momento actual. Respalda lo anterior el hecho de que nadie entre los directores o directoras obtuvo puntuaciones que se correspondan con el nivel alto en los componentes cansancio emocional y despersonalización, y tampoco se observaron casos de realización personal en un nivel bajo.

\section{Análisis descriptivo}

Para profundizar el análisis de los resultados obtenidos, y en el entendido de que los directores o directoras que padecen burnout en algún grado no necesariamente presentan los mismos síntomas y evolución, se realiza un análisis de las puntuaciones que alcanzaron los 22 sujetos directores de instituciones educativas públicas de la zona de Barrios Altos, Lima, categorizando los datos en función de una serie de factores más importantes que explican la prevalencia del síndrome de burnout.

\section{a) Sexo}

Es un factor presente en la inmensa mayoría de los estudios psicométricos. Si bien es cierto que el personal en funciones de dirección escolar de las instituciones educativas de Barrios Altos está conformado en su mayoría por mujeres ( 14 de sexo femenino, 8 de sexo masculino), lo cual dificulta en cierta medida hacer comparaciones, los datos obtenidos muestran una leve, pero significativa tendencia de las directoras a tener mayor cansancio emocional. Este resultado se corresponde con las obtenidas en otros estudios sobre burnout (Maslach y Jackson, 1986), pero no coincide completamente, pues en el sexo femenino se encontró también mayor despersonalización. El $85,7 \%$ del total de directoras posee un nivel medio de cansancio emocional, mientras que en los directores con nivel medio se reduce a $62,5 \%$. Asimismo, en el componente realización personal nivel alto, también se aprecia una diferencia, siendo 50\% en las mujeres y $75 \%$ en los hombres.

\section{b) Edad}

La edad es una variable que tiende a ser considerada para estudio en correlación con el burnout, pues la persona trabajadora experimentaría una mayor vulnerabilidad y procesos particulares propios de cada etapa de su vida, sin embargo, no hay estudios concluyentes al respecto. En este estudio, los resultados no muestran que la edad constituya un factor que muestre variaciones tan significativas que permitan ubicarlo como un factor que ejerce influencia en la prevalencia del burnout en la dirección escolar pública de la zona de Barrios Altos, pues cada segmento de edad tiende a reproducir el patrón obtenido en los resultados por componentes: cansancio emocional (medio), despersonalización (bajo) y realización personal (alto). 
http://doi.org/10.15359/ree.25-3.28

http://www.una.ac.cr/educare educare@una.ac.cr

\section{c) Experiencia en la dirección de la institución educativa}

La experiencia o tiempo de servicio en determinada función o cargo constituye un factor también tomado en cuenta a la hora de hacer estudios descriptivos sobre el burnout. En cuanto a la incidencia del factor experiencia en el cargo de dirección en los resultados de la investigación, se mantiene el patrón de puntuaciones por componentes: cansancio emocional (medio), despersonalización (bajo) y realización personal (alto).

La única variación en el patrón de puntuaciones antes mencionado se encuentra en el personal directivo que tiene entre seis a diez años de experiencia, que obtuvo puntuaciones en despersonalización de nivel medio $(71,4 \%)$, y realización personal también medio $(85,7 \%)$. Estos datos Ilevan a advertir que en este segmento de entre 6 y 10 años de experiencia directiva hay una posible predisposición hacia el síndrome de burnout.

\section{Discusión}

El caso del personal que realiza funciones de dirección escolar en la zona de Barrios Altos es digno de estudio, por cuanto desempeñan sus labores en un espacio territorial que tiene una gran relevancia histórica y cultural de la ciudad de Lima, pero que presenta una gran cantidad de problemas sociales asociados a la pobreza que, indefectiblemente, tienen un impacto en la dinámica laboral de las instituciones educativas públicas.

En este sentido, se consideró estudiar a la totalidad de los directores y directoras escolares de las instituciones públicas, colectivo integrado por 22 profesionales, y aunque se tomó el $100 \%$ de la población a estudiar, se trata de una muestra relativamente pequeña desde el punto de vista estadístico $(\mathrm{N}=22)$. Por esta razón, los resultados obtenidos tienen una finalidad descriptiva, sin vocación de generalización.

Los resultados de la aplicación del Maslach Burnout Inventory (Maslach y Jackson, 1981, 1986) refieren un nivel medio de agotamiento emocional, bajo nivel de despersonalización y alta realización personal. Esto lleva a inferir que la prevalencia del síndrome de burnout en este colectivo es baja, por cuanto los estudios señalan como indicadores de burnout alto agotamiento emocional, alta despersonalización y baja es la realización personal de burnout (Cooper y Kelly, 1993; Lainas, 2010; Maslach y Jackson, 1981. El estudio refleja la presencia de agotamiento emocional en forma media, y podría considerarse que existen indicios para afirmar que en el colectivo con una experiencia directiva que oscila entre seis y diez años podría haber una predisposición hacia el síndrome de burnout. 
http://doi.org/10.15359/ree.25-3.28

http://www.una.ac.cr/educare

educare@una.ac.cr

\section{Conclusiones}

Dirigir una institución educativa es una tarea altamente exigente, debido a que el éxito depende no solo de las habilidades y competencias de quien dirige, sino de la sumatoria de las características personales del equipo, del contexto en que está inmersa, y la política educativa. Ante la diversidad de roles de la función directiva en la actualidad, las nuevas demandas y la creciente presión sobre la figura directiva, ejercer esta labor conlleva una continua exposición a estresores, que, si no se manejan adecuadamente, podrían desencadenar en el síndrome de burnout u otras patologías (Chaplain, 2001).

Si bien es cierto que la bibliografía refiere que el origen del síndrome de burnout se encuentra en unas elevadas demandas laborales (Lee y Ashforth, 1996), sin embargo, identificar con exactitud sus factores desencadenantes constituye una tarea compleja. Con el Maslach Burnout Inventory es posible estimar la prevalencia del burnout, analizando los niveles de agotamiento emocional, despersonalización y realización personal. Sin embargo, es importante tener presente que el Maslach Burnout Inventory tiene como foco de atención la dimensión emocional, y sin pretender cuestionar su validez y propiedades psicométricas, se requiere complementarlo con otras herramientas de naturaleza interpretativa o cualitativa, que complementen el estudio, de cara a la complejidad que supone el síndrome.

En cuanto a la prevalencia del síndrome de burnout en la dirección escolar de las instituciones educativas públicas de la zona de Barrios Altos, Lima, los resultados dan cuenta de una baja probabilidad de que este fenómeno esté afectando al personal en funciones de dirección escolar, pero sí muestra un agotamiento emocional en nivel medio, y podría considerarse que existen indicios para afirmar que a directores y directoras con entre seis y diez años de experiencia directiva podría haber una predisposición hacia el síndrome de burnout. Estos resultados obtenidos sostienen la necesidad de que el personal en funciones de dirección escolar en Barrios Altos reciba herramientas cognitivas y motivacionales que les permitan manejar el agotamiento emocional que se refleja en el estudio, y que puede encontrar explicación en las desfavorables condiciones en las que desempeñan sus labores directivas.

Este estudio constituye un aporte investigativo preliminar, que deja el camino abierto para la realización de otros relacionados con la dirección escolar y el síndrome de burnout en la zona de Barrios Altos, Lima, y en otros contextos. El importante papel de la dirección escolar para el logro de una educación con crecientes niveles de calidad requiere, además, que promueva el mejoramiento de sus condiciones laborales, para minimizar o manejar de una manera más efectiva la exposición a los estresores propios de los contextos de vulnerabilidad social.

Desde la academia y las diversas instancias del gobierno local, regional y nacional se deben realizar nuevas investigaciones acerca de la dirección escolar y el síndrome de burnout, las cuales, para que tengan un impacto real, deben traducirse en acciones transformadoras, con efectos reales en la calidad educativa. 


\section{Declaración de Material complementario}

Este artículo tiene disponible, como material complementario:

-La versión preprint del artículo en https://doi.org/10.5281/zenodo.4890965

\section{Referencias}

Alsalamah, A., y Callinan, C. (2021). Adaptation of kirkpatrick's four-level model of training criteria to evaluate training programmes for head teachers. Education Sciences, 11(3), 1-25. http://dx.doi.org/10.3390/educsci11030116

Barroso, J. (2005). Liderazgo y autonomía de los centros educativos. Revista Española de Pedagogía, 63(232), 423-442. https://revistadepedagogia.org/wp-content/uploads/2007/06/232-07.pdf

Bolívar, A. (2015). Evaluar el liderazgo pedagógico de la dirección escolar. Revisión de enfoques e instrumentos. Revista Iberoamericana de Evaluación Educativa, 8(2), 15-39. https://revistas. uam.es/riee/article/view/2740/2927

Chaplain, R. P. (2001). Stress and job satisfaction among primary headteachers. A question of balance. Educational Management Administration \& Leadership, 29(2), 197-215. https://doi. org/10.1177/0263211X010292005

Cooper, C. L. y Kelly, M. (1993). Occupational stress in headteachers: A national UK study. British JournalofEducationalPsychology,63(1),130-143.https://doi.org/10.1111/j.2044-8279.1993. tb01046.x

Freudenberger, H. J. (1974). Staff burn-out. Journal of Social Isssues, 30(1), 159-165. https://doi. org/10.1111/j.1540-4560.1974.tb00706.x

Friedman, I. A. (1995). School principal burnout. The concept and its components. Journal of Occupational Behaviour, 16(2), 191-198. https://doi.org/10.1002/job.4030160209

Fullan, M. (2014). The principal: Three keys to maximizing impact. Jossey-Bass.

Gil-Monte, P. R. (2002). Validez factorial de la adaptación al español del Maslach Burnout Inventory-General Survey (MBI-GS) en una muestra de policías municipales. Salud Pública de México, 44(1), 33-40. https://doi.org/10.1590/\$0036-36342002000100005

Gil-Monte, P. R. (2003). Burnout syndrome: ¿Síndrome de quemarse por el trabajo, desgaste profesional, estrés laboral o enfermedad de Tomás? Revista de Psicología del Trabajo y de las Organizaciones, 19(2), 181-197. https://www.redalyc.org/pdf/2313/231318052004.pdf

Gil-Monte, P. R. (2006). El síndrome de quemarse por el trabajo (Burnout): Una enfermedad laboral en la sociedad del bienestar. Ediciones Pirámide. 
http://doi.org/10.15359/ree.25-3.28

http://www.una.ac.cr/educare

educare@una.ac.cr

Gil-Monte, P. R. y Peiró, J. M. (1997). Desgaste psíquico en el trabajo: El síndrome de quemarse. Síntesis.

Golembiewski, R.T., Scherb, K.y Boudreau, R. A. (1993). Burnout in cross-national settings: Generic and model-specific perspectives [Burnout en entornos transnacionales: Perspectivas genéricas y específicas del modelo] En W. B. Schaufeli, C. Maslach, y T. Marek (Eds.), Professional burnout: Recent developments in theory and research (pp. 217-236). Taylor and Francis. https://doi.org/10.4324/9781315227979-17

Hakan, S. (2004) An analysis of burnout and job satisfaction among Turkish special school headteachers and teachers, and the factors effecting their burnout and job satisfaction. Educational Studies, 30(3), 291-306. https://doi.org/10.1080/0305569042000224233

Hederich-Martínez, C. y Caballero-Domínguez, C. C. (2016). Validación del cuestionario Maslach Burnout Inventory-Student Survey (MBI-SS) en contexto académico colombiano. Revista CES Psicología, 9(1), 1-15. http://www.scielo.org.co/pdf/cesp/v9n1/v9n1a02.pdf

Hughes, M. (1987). Leadership in professionally staffed organizations. En M. Hughes, P. Ribbins y H. Thomas (Eds.), Managing education: The system and the institution. (pp. 3-43). Cassell.

Kulakova, O., Moreno Jiménez, B., Garrosa, E., Sánchez Hernández, M. O. y Aragón, A. (2017). Universalidad del constructo del Maslach Burnout Inventory en un contexto latinoamericano. Acta de Investigación Psicológica, 7(2), 2679-2690. https://doi. org/10.1016/j.aipprr.2017.05.001

Lainas, A. (2010). Local directors of school education in Greece: Their role in main sources of job stress. Educational Management Administration \& Leadership, 38(4), 454-471. https://doi. org/10.1177/1741143210368145

Lee, R. T. y Ashforth, B. E. (1996). A meta-analytic examination of the correlates of the three dimensions of job burnout]. Journal of Applied Psychology, 81(2), 123-133. https://doi. org/10.1037/0021-9010.81.2.123

Louis, K. S. y Miles, M. B. (1991). Improving the urban high school: What works and why. NASSP Bulletin, 75(534), 117-119. https://doi.org/10.1177/019263659107553420

Martínez-Garrido, C. (2017). La incidencia del liderazgo y el clima escolar en la satisfacción laboral de los docentes en América Latina. Archivos Analíticos de Políticas Educativas, 25(80), 1-22. https://doi.org/10.14507/epaa25.2851

Maslach, C. (1976). Burned-out. Human Behavior, 9(5), 16-22. https://www.researchgate.net/ publication/263847499 Burned-Out

Maslach, C. (2017). Finding solutions to the problem of burnout. Consulting Psychology Journal: Practice and Research, 69(2), 143-152. https://doi.org/10.1037/cpb0000090 
http://doi.org/10.15359/ree.25-3.28

Maslach, C., Schaufeli, W. B. y Leiter, M. P. (2001). Job burnout. Annual Review of Psychology; 52, 397- 422. https://doi.org/10.1146/annurev.psych.52.1.397

Maslach, C. y Jackson, S. E. (1981). The measurement of experienced burnout. Journal of Occupational Behaviour, 2(2), 99-113. https://doi.org/10.1002/job.4030020205

Maslach, C. y Jackson, S. E. (1986). Maslach Burnout Inventory: Manual (2. ${ }^{a}$ ed.). Consulting Psychologists Press.

Olayiwola, S. (2008). Dimensions of job stress among public secondary school principals in Oyo state, Nigeria. https://files.eric.ed.gov/fulltext/ED503654.pdf

Ramery-Gelpi, E. y Pérez Navío, E. (2016). Comparación de la satisfacción laboral del director escolar y los docentes. Revista Electrónica de Investigación y Docencia, 15, 85-100. https:// doi.org/10.17561/reid.v0i15.2797

Sáenz Barrio, Ó. y Debón Lamarque, S. (1995). Teorías sobre el deterioro de la dirección escolar. Revista Interuniversitaria de Formación del Profesorado, 24, 193-206. https://dialnet.unirioja. es/servlet/articulo?codigo $=117888$

Tahira, B. y Afshan, H. (2019). The antecedents of burnout in heads of secondary schools of Khyber Pakhtunkhwa: A study of relationships among burnout, organizational commitment and emotional intelligence. Global Regional Review, 4(4), 168-177. https://doi.org/10.31703/ grr.2019(IV-IV).19

Tejero González, C. M., Fernández Díaz, M. J. y Carballo Santaolalla, R. (2010). Medición y prevalencia del síndrome de quemarse por el trabajo (burnout) en la dirección escolar. Revista de Educación, 351, 361-383. http://www.educacionyfp.gob.es/revista-deeducacion/numeros-revista-educacion/numeros-anteriores/2010/re351/re351-15.html

Veenman, S. (1997). El director de centros docentes como formador. En Villa, A. (Coord.). Dirección participativa y evaluación de centros (pp. 557-578). Publicaciones de la Universidad de Deusto.

Xu, Z., y Yang, F. (2021). The impact of perceived organizational support on the relationship between job stress and burnout: A mediating or moderating role. Current Psychology, 40(1), 402-413. https://doi.org/10.1007/s12144-018-9941-4

Yusof, A. (2012). Going to bed with your work: Head teachers' burnout and sleep quality. International Journal of Economics Business and Management Studies, 1(1), 1-8. https://ssrn. com/abstract $=2150971$ 